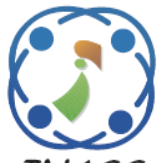

\title{
Inflation Rate Forecasting Using Extreme Learning Machine and Improved Particle Swarm Optimization
}

\author{
Wayan Firdaus Mahmudy ${ }^{1 *}$ \\ Adyan Nur Alfiyatin $^{1} \quad$ Candra Fajri Ananda ${ }^{2}$ \\ ${ }^{I}$ Faculty of Computer Science, Universitas Brawijaya, Indonesia \\ ${ }^{2}$ Faculty of Economy and Business, Universitas Brawijaya, Indonesia \\ * Corresponding author’s Email: wayanfm@ub.ac.id
}

Agus Wahyu Widodo ${ }^{1}$

\begin{abstract}
Inflation is an important tool to assess the current condition of a nation economy. Uncontrolled inflation rate may have a lot of negative impacts on economic development. Forecasting can be used to control the inflation by making appropriate economic policies. However, uncertainty pattern of the inflation rate may make it hard to forecast. This study proposes an extreme learning machine (ELM) for the inflation rate forecasting. As part of the machine learning algorithm, the ELM has an ability to address uncertainty in data input pattern. However, ELM had weakness in determining initial weights that may produce inaccurate results. So, we propose particle swarm optimization (PSO) to determine good initial weights for the ELM. PSO is a metaheuristic method that gives good results in local searches but requires longer computation time to locate its particles on the global optimum point in the vast search space area. To overcome this problem, auto-speed acceleration algorithm is employed to drive particles of the PSO in searching of the global optimum with lower computation time. The performance of the proposed approach is evaluated using root mean square error (RMSE). A series of computational experiments prove that the proposed approach achieves better results with the average RMSE of 0.01926. This result is better than RMSE of 0.02020 achieved by the original version of ELM.
\end{abstract}

Keywords: Inflation rate, Forecasting, Extreme learning machine, Particle swarm optimization, Auto-speed acceleration algorithm.

\section{Introduction}

Inflation is one of the macroeconomic variables that influences the stability of a country's economy [1]. The economy is said to be stable if the impact of a turmoil shows relatively small changes to macroeconomic variables and does not require a long time to achieve long-term balance. On the contrary, the state economy is said to be unstable and vulnerable to change if the impact of a shock causes large fluctuations in macroeconomic variables and requires a relatively long time to achieve long-term balance.

In Indonesia, to maintain and improve economic growth stability, the monetary policy is managed directly by Bank Indonesia [2]. Bank Indonesia implemented inflation targeting framework to keep inflation low and stable. Inflation comes from two factors, they are domestic and abroad pressures [3]. Domestic pressures are everything that happens in the country that affects the price of goods. Abroad pressures such as inflation in other countries that will affect exports, imports, or balance of payments between countries, the increase in prices of imported goods will cause an increase in product prices with imported raw materials. If the imported goods are very essential needs to the importing country, then the increase of these imported goods affect domestic inflation pressures quite significantly. Therefore, they will have a high impact on price changes. To control inflationary pressures from both domestic and abroad, the Indonesian government must use the right strategy to make inflation runs stable. Inflation rate forecasting is required to help the government formulating appropriate policies for the economic development. 
The fluctuate pattern of the inflation rate and numerous variables that may influence the rate make the forecasting is a challenging task. Thus, a powerful method is required for the inflation rate forecasting. The method should address uncertainty patterns that often occur in the inflation rate trends.

Statistical based methods are widely used for forecasting task [4]. The methods include Adaptive autoregressive moving average (ARMA) and auto regressive integrated moving average (ARIMA) that requires only historical data [5]. Even though the statistical based methods could provide accurate results in several studies, they have limitations to address some patterns in financial time series data such as sudden changes in data pattern on irregular time interval [6].

Many current studies found that artificial intelligence based algorithms provide better accuracy for forecasting [7]. The methods include artificial neural network based methods [8] and fuzzy logic based methods [9]. For inflation rate forecasting, many methods have been used such as backpropagation neural network [10], fuzzy neural system [11], extreme learning machine [12], Hybridizes Fuzzy and PSO-auto speed acceleration algorithm [13], Hybrid ELM and particle swarm optimization [14], Hybrid ELM and genetic algorithms [14]. While the studies report promising result, there are opportunity to do a similar study by implementing a more powerful approach with higher accuracy and lower computational time. The backpropagation neural network will be used as a baseline method in Section 3 to prove the effectiveness of the proposed approach. The other methods will be examined their advantages and limitations in the rest of this section.

The inflation rate forecasting performed by Sari et al. [10] uses data derived from external factors including time series inflation data, CPI, money supply, exchange rate and BI rate. The method of backpropagation get an RMSE value of 0.204 then the research is continued using the method FNS (Sugeno fuzzy reference system and Backpropagation Neural Network) to get a smaller error value and speed up the computation time, but this research gets a larger RMSE value there is 2.154901 [15]. The other limitation is the backpropagation neural network require a high computational time to obtain the best rule for Sugeno fuzzy reference system.

Further research is carried out by Alfiyatin et al. [13] by adding external factors such as time series inflation data, CPI, money supply, exchange rate, BI rate, GDP (total credit and total assets data) using the ELM method which has fast computing time because there are no iterations in the training and testing process, value the resulting error is 0.0202008 smaller than the previous study even though with the addition of the input variable. Thus, it is proving that ELM is able to solve more complex problems with a short computation time. However, ELM had weakness in determining initial weights that may produce inaccurate results. The ELM is also used as a comparative method in this study.

The next inflation rate prediction uses a hybridization between the Fuzzy method and the PSO-Auto Speed Acceleration Algorithm, the data used includes time series inflation data, CPI, money supply, exchange rate, and BI rate. The study proposes an improved PSO method in which PSO is good in the local search process and has not been optimal in global search, so with the addition of a setting on the PSO interval called an auto-speed acceleration algorithm, it is hoped that PSO will be one of the best metaheuristic methods in the particle search process both local area and global area. The RMSE generated in this study is 0.106263822 [16]. While the computational time is quite low, the RMSE is larger than results of other approaches.

In their research, Alfiyatin et al. [14] combine the ELM method optimized using particle swarm optimization and ELM optimized using genetic algorithm. ELM is good for solving forecasting problems with fast training times, able to solve complex problems and can be applied in real life and have a clear hidden node mapping on feature mapping. In addition to the advantages, ELM also has disadvantages that the resulting accuracy value is uncertain because the weight and bias values are determined randomly, so a metaheuristic method is needed to determine weights and bias in order to get better results. The research used time series inflation data, CPI, money supply, exchange rate, BI rate, GDP (data on total credit and total assets) get RMSE values of 0.022081054 and 0.020202758 . To prove the effectiveness of our proposed method, the ELM optimized using particle swarm optimization and ELM optimized using genetic algorithm are also used as comparative method in in this study.

Some previous studies stated that the extreme learning machine (ELM) method obtained the best accuracy results compared to the other methods [14], $[17,18]$. The ELM also required lower computational time. However, the ELM produces fluctuating results due to the determination of the weight value and the bias is done randomly so that additional methods are needed for determining the appropriate weights and biases [19]. Based on the previous studies, the addition of optimization methods for determining weight and bias can 
effectively increase the performance of ELM, fewer errors were obtained and more accurate results [20].

PSO is a metaheuristic method that gives good results in local searches, and requires a long computation time for the search process for particles in a global area [14]. In the search process for global area solutions to obtain maximum results, it is necessary to add optimum interval settings to speed up computation time $[16,21]$. The method used for the optimum interval setting is the auto-speed acceleration algorithm, which is inspired by the concept of one-dimensional motion in classical physics developed by Galileo Galilei and Isaac Newton. [22]. One-dimensional motion is a particle that moves from one position to another position and is affected by acceleration. Particle acceleration affects velocity so that it can control the distance the particles move. Thus, the auto-speed acceleration algorithm can control particle displacement to find optimal solutions [16].

Apart from particle displacement, the determination of particle motion also greatly influences the particle search process so that it can get out of local searches to find optimal solutions. The auto-speed acceleration algorithm can quickly move to the solution points because it can adjust the range of particle displacement. In addition, the autospeed acceleration algorithm only requires primitive mathematical operators on one-dimensional kinetic motion, so it only adds a few lines of simple computer program code. The focus of the auto-speed acceleration algorithm is to make adjustments to the speed of motion of each particle moving process which will affect changes in speed, direction and distance $[16,22]$.

To sum up, the inflation rate forecasting is a challenging task and requires a powerful method to address the fluctuate pattern of the inflation rate historical data. The extreme learning machine (ELM) method obtained the best accuracy results for forecasting compared to the other methods and also requires a lower computational time. As the ELM had weakness in determining initial weights that may produce inaccurate results, the particle swarm optimization (PSO) is employed to determine good initial weights for the ELM. The auto-speed acceleration algorithm is employed to drive particles of the PSO in searching of the global optimum with lower computation time.

This research will show the results of implementing the ELM and PSO-auto speed acceleration algorithm to forecast the inflation rate in Indonesia. Comparative methods that will be used to prove research results include backpropagation, extreme learning machines, Hybrid ELM and genetic algorithms, Hybrid ELM and particle swarm optimization by displaying error values using RMSE.

This paper consists of four sections. Section 1 presents an introduction that explains the background problem and the need for more accurate method. Previous related works that are used for inflation rate forecasting are also discussed in this section. The development of the proposed approach is presented in Section 2. Section 3 discusses computational experiment results and the comparison with other methods. Conclusions are presented at the last section.

\section{The proposed method}

This research used the hybridization of extreme learning machine and PSO-auto speed acceleration algorithm. The use of PSO - auto speed acceleration algorithm method to obtain the best weight value that will be used in the ELM method in order to obtain optimal results. The hybrid model will fully

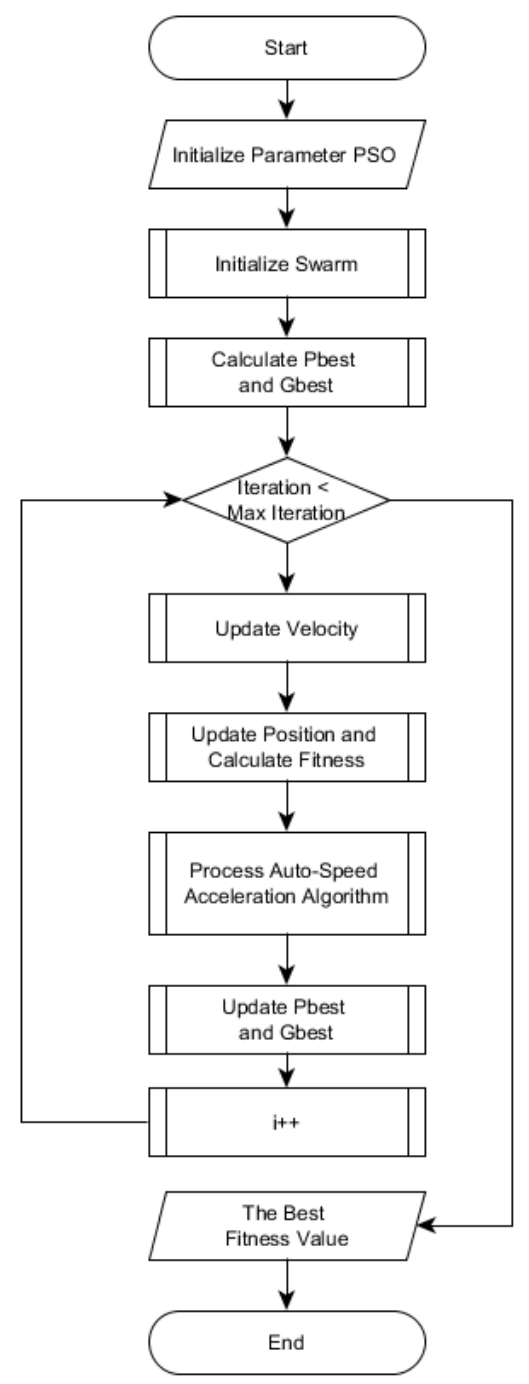

Figure. 1 Flowchart of the proposed method 
utilize the strong advantages of each method. Thus, the main contribution of this study is providing an effective mechanism to improve the performance of the ELM using PSO-auto speed acceleration algorithm. In this section, we will show how the auto speed acceleration algorithm empower the PSO to move its particles for searching the global optimum. The best particle of the PSO will be converted into weights of the ELM for accurate forecasting.

Fig. 1 shows the hybridization flowchart of the proposed method.

The detailed steps in each method are as follows:

\subsection{PSO-auto speed acceleration algorithm}

Particle Swarm Optimization was developed by Kennedy and Eberhart [23]. PSO is inspired by the social behavior of animals, it is a group of birds in a swarm. PSO is a metaheuristic method and begins with a random population called particles. In the $\mathrm{PSO}$, process is related to a velocity and the velocity moves through a dynamic velocity space search according to its historical behavior. The flow of the PSO method includes particle initialization is carried out randomly from 0 to 1 , update velocity, update PBest (local fitness) and Gbest (global fitness) and calculate the fitness value.

Auto-speed acceleration algorithm is inspired by the concept of motion in one dimension where the motion is influenced by acceleration and time for the displacement of position on particles, this method based on Galileo Galilei and Isaac Newton[22]. The auto-speed acceleration algorithm uses fundamental math so just only need to add a few simple lines of code to do the process. The auto-speed acceleration algorithm has 3 components that influence each other, namely changes in speed, direction and distance [16]. Fig. 2 will explain the flow of the auto-speed acceleration algorithm.

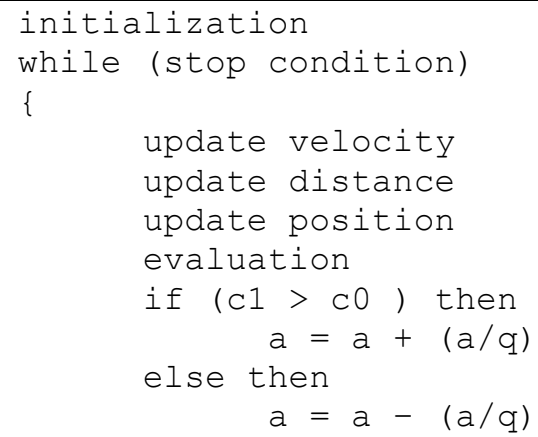

Figure. 2 Pseudo-code auto-speed acceleration algorithm
Fig. 2 shows the pseudo code auto-speed acceleration algorithm. Study [16] explained that if PSO is added with an auto-speed acceleration algorithm, the weakness of PSO will be resolved, PSO will be good in local searches and global searches with short computation time.

\subsection{Extreme learning machine (ELM)}

ELM belongs to artificial neural networks learning method that was introduced by Huang et al. [24]. ELM has only single hidden layer in its network architecture, so it is commonly called the Single-Hidden Layer Feedforward Neural Network. This method is used to correct the previous method, for example, the back propagation method. Improvements in learning-speed from the learning process [25]. The steps of ELM are normalization data, training and testing process, and denormalization data.

Training process consist several steps as follow:

1. Initialize the input weight and bias. This value is initialized randomly between the values 0 to 1 .

2. The hidden layer output is calculated using the activation function. The first step is to calculate the hidden layer output $\left(\mathrm{H}_{\text {init }}\right)$, After getting the $\mathrm{H}_{\text {init }}$ value, Eq. (1) will show the equation for calculating the hidden layer output:

$$
H_{\text {init } i j}=b_{j}+\sum_{i=1}^{n}\left(x_{i} w_{j i}\right)
$$

$$
\begin{aligned}
& i=[1,2, \ldots \mathrm{N}] . \mathrm{N} \text { is total data. } \\
& j=[1,2, \ldots \mathrm{N}] . \mathrm{N} \text { is total hidden neuron. } \\
& n=\text { total input neuron } \\
& w=\text { input weight } \\
& x=\text { input data } \\
& b=\text { bias value }
\end{aligned}
$$

3. The third stage is to find the hidden layer output matrix by calculating the results from the $H_{\text {init }}$ with the sigmoid activation function $(H[x])$.

4. The fourth stage is to transpose the hidden layer output matrix with the sigmoid activation function $\left(H^{T}\right)$.

5. The fifth stage is to multiply the hidden layer output with the sigmoid activation function $\left(H^{T}\right)$ with a hidden layer output matrix with the sigmoid activation function $(H(x))$.

6. After the fifth stage has been completed, the matrix from the multiplication is inverted and then the inverse matrix results are multiplied by the hidden layer output matrix with the transposed sigmoid activation function. The output of this multiplication is called Moore- 
Penrose Generalized Inverse $\left(\mathrm{H}^{+}\right)$according to the Eq. (2).

$$
H^{+}=\left(H^{\mathrm{T}} H\right)^{-1} H^{\mathrm{T}}
$$

7. The seventh step is to calculate the output weight. The steps in calculating the output weight are in accordance with Eq. (3).

$$
\beta=H^{+} T
$$

$$
\begin{aligned}
& \beta=\text { output weight matrix } \\
& H+=\text { Moore-Penrose Generalized of Matrix } \\
& \quad \text { Invers from matrix } \mathrm{H} \\
& T=\text { target matrix }
\end{aligned}
$$

\subsection{Comparion methods}

Several approaches, backpropagation neural network, ELM, hybrid ELM and PSO, and hybrid ELM and genetic algorithm, are used as comparison methods to prove the effectiveness of hybrid ELM, PSO, and auto-speed acceleration algorithm. The ELM and the PSO have been explained in the previous sub-section as parts of the proposed approach. In this sub-section we explain details of the backpropagation neural network and the genetic algorithm.

The backpropagation algorithm is a method to train an artificial neural network. The algorithm systemically update weights of the artificial neural network so targeted outputs are obtained. In this study, the targeted outputs are forecasting result. The algorithm starts by generating random initial weights. The weights are used to calculated outputs the artificial neural network. Output errors are calculated by comparing the artificial neural network outputs and the targeted outputs. The errors are used to adjust the weights so lower errors will be obtained in the next iteration. The iteration is repeated until the output error is less than predetermined threshold. Detail of the algorithm can be found in [26].

The genetic algorithm is a popular optimization technique that works by imitating natural evolution. In this study, the genetic algorithm is used as comparison method to optimize the initial weights of the ELM. The genetic algorithm works by generating initial populations that contain a number of chromosomes, The chromosome is representation of a solution. The chromosome is evaluated using a fitness function to measure its appropriateness as a candidate solution. Using several genetic operators, new chromosomes are produced in each generation. The best chromosome in the last generation is chosen as the solution. Detail of the algorithm can be found in [27].

\section{Result and discussion}

In this study, to determine the accuracy of the proposed method, the researcher compared several methods such as backpropagation, extreme learning machine (ELM), Hybrid ELM and PSO, and Hybrid ELM and genetic algorithms. This section will discuss the tests carried out on the proposed method and the tests will use the best parameters of the PSO-ELM model [14]. Because the proposed method is an improved particle swarm optimization method. PSO is good for local searches [28], while the addition of an auto-speed acceleration algorithm for global search [29]. The improvement aims to get optimal value both locally and globally. This study employs 100 particles and 110 iterations. The value of inertia weight is 0.8 and acceleration coefficient is set to 2. The number of neurons in the hidden layer is set to 3 . The data used in the training stage were 109 data and 47 data testing stages. Tests carried out include testing the variable of acceleration $(r)$ and testing number of times $(t)$.

This study use data from Bank Indonesia and BPS from January 2005 to December 2017. The parameters used for inflation forecasting consist of CPI, interest rates, money supply, exchange rate, credit value, asset value and time series data (inflation data for the previous 1 month [y-1], two months before [y-2] and 3 months before [y-3]. The parameters are used as input variables and the output variables are the results of the forecasting of inflation rate in Indonesia.

\subsection{Particle number testing}

The first test that is carried out is the particle test, to find the most appropriate number of particles that will be used in the system in order to obtain the best solution. In this test, the interval of values used is 10-150 with multiples of 10 for each number of particles. The other parameters values are iterations of 100 , inertia weight of 0.6 and acceleration coefficient $(c 1)$ of 2 , and $c 2$ of 1 . The range used in the particle count test is narrower because PSO is better for small area searches [14].

Fig. 3 shows that the RMSE is continuously reduced until the number of particles reaches 40 . A slightly fluctuated values of RMSE are obtained for the higher of particles number. The lowest RMSE value is obtained for number of particles of 110 . This value will be used for the next test. 


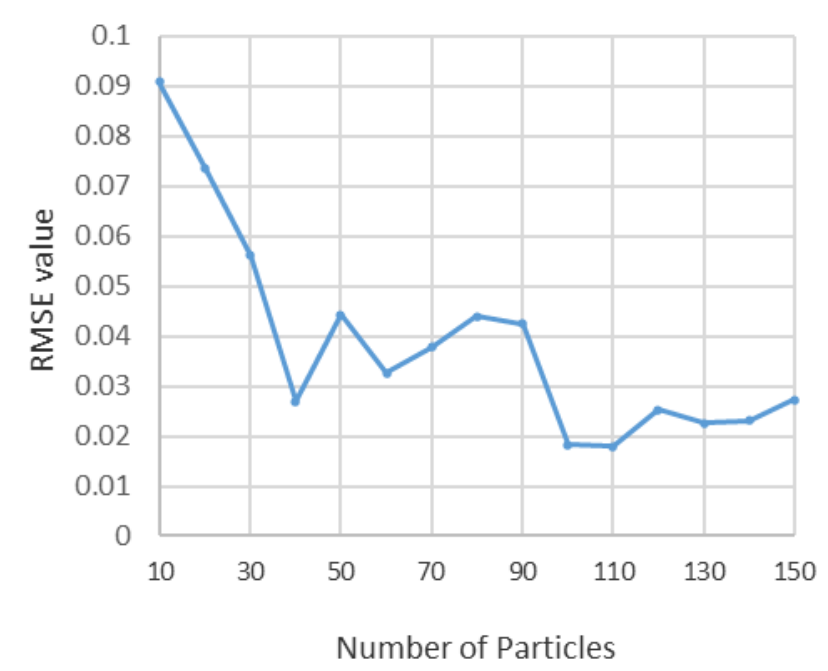

Figure. 3 Result of the number particle testing

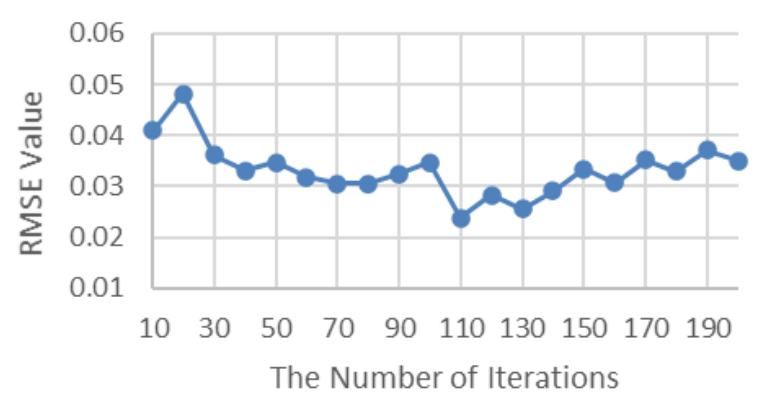

Figure. 4 Result of the number iterations testing

\subsection{Number of iterations testing}

Testing the number of iterations aims to determine the best number of iterations to obtain the lowest value of RMSE. A lower number of iterations will not enable the PSO to show its best performance. In contrast, a higher number of iterations will require a high computational time. The iteration value tested is from 10 to 200 with multiples of 10 . Other parameter values to be used are the number of particles as much as 110 , the inertia weight of 0.6 and the acceleration coefficient of 2 and 1 for $c_{1}$ and $c_{2}$ respectively.

Fig. 4 shows that the lowest RMSE value is obtained in the number of iterations of 110 . As the pattern in the number of particles test, there is no significant reduction of the RMSE. Thus, we decide that the best number of iterations is 110 . This value will allow the PSO exploring the search in reasonable amount of time.

\subsection{Inertia weight testing}

The next step is inertia weight $(w)$ testing. The test is required to determine the best value of inertia weight that produce the lowest RMSE. The value of inertia weight is set starting from 0 to 1 in

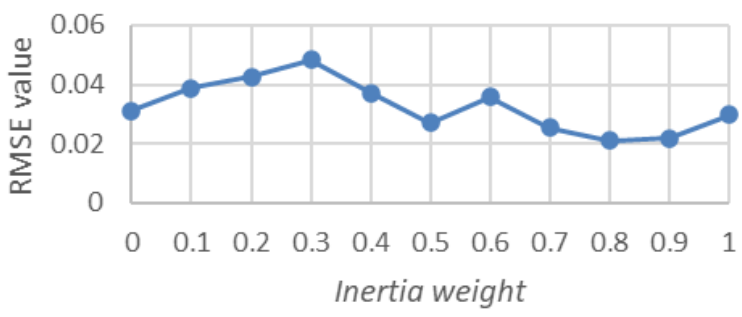

Figure. 5 Result of inertia weight testing

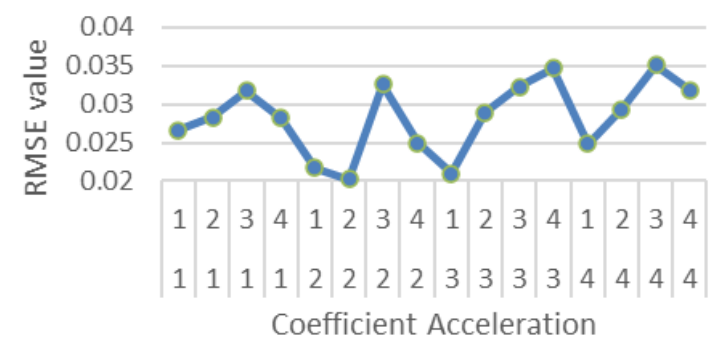

Figure. 6 Result of coefficient acceleration testing

increments of 0.1 and generally the best range value lies at the point 0.4 to 0.9 [30]. Another parameter used is the number of particles of 100 , iteration $=$ $110, c_{1}=2$ and $c_{2}=1$. The results of this test will be used in the next test.

Fig. 5 shows that the best inertia weight value is 0.8 . This finding is confirmed by [31] that states the best $w$ value is in range 0.5 and 1 . If $w$ is greater than 1 the velocity become uncontrolled and unstable particles movement is obtained. In contrast, lower $w$ value will make the particles of PSO slowly move in the search space area.

\subsection{The acceleration coefficient testing}

The acceleration coefficient testing is done to determine the particles movement pattern in one iteration. This test is carried out using the same scenarion as in [32]. The test result is provided in Fig. 6.

As shown in Fig. 6, the lowest RMSE is obtained if both $c_{1}$ and $c_{2}$ equal to 2 . The values of $c_{1}$ and $c_{2}$ influence the direction of motion of a particle to the local best or the global best [31].

\subsection{The number of neurons testing}

Test in this stage is carried out to determine the number of neurons in the hidden layer of ELM. The test scenario is adopted from [33] that assume the greater the number of neurons used, the more complex it is in determining the best neuron weights and also requires a long computation time.

Fig. 7 shows that the RMSE is slightly reduced with the addition of the number of neurons in the hidden layer. This can occur because the greater 
Table 1. The number of hidden neurons and computing time

\begin{tabular}{|c|c|c|c|c|c|}
\hline \multirow{3}{*}{ nh } & \multicolumn{4}{|c|}{ particles 100, iteration $110, c 1 \&$} & \multirow{2}{*}{$\begin{array}{c}\text { Time } \\
\text { (seconds) }\end{array}$} \\
\cline { 2 - 5 } & Test1 &.. & Test6 & Avg & \\
\hline 3 & 0.0197 &.. & 0.0127 & 0.01902 & 9 \\
\hline 4 & 0.0126 &.. & 0.0143 & 0.01802 & 35 \\
\hline 5 & 0.0174 &.. & 0.0105 & 0.01710 & 116 \\
\hline 6 & 0.0288 &.. & 0.0151 & 0.01705 & 727 \\
\hline 7 & 0.0127 &.. & 0.0174 & 0.01664 & 6,041 \\
\hline 8 & 0.0111 &.. & 0.0174 & 0.01664 & 66,242 \\
\hline
\end{tabular}

nh: number of hidden neurons

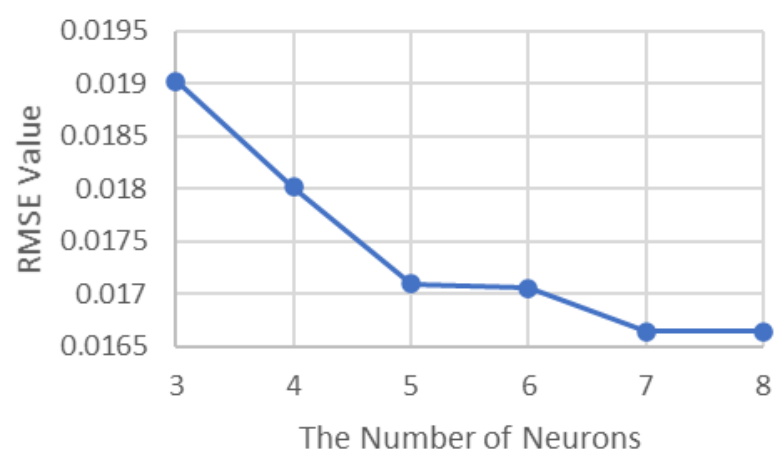

Figure. 7 Result of the number of hidden neuron testing

the number of neurons, the more complex the mathematical equation will be and produce a better value than the small number of neurons, but it requires a longer computation time. This is consistent with the research of Huang and Lai [34] which states that the more the number of neurons used, the greater the accuracy value obtained and requires longer computation time. In this study, 3 neurons are used with the consideration that the computation time is shorter and the error values obtained are not much different from other values.

Based on all the tests that have been done, we set the PSO-ELM architecture for forecasting the inflation rate with the optimal results using 100 particles, 110 iterations, inertia weight of 0.8 , acceleration coefficient equal to 2 and the number of neurons of 3 .

\subsection{Variables of acceleration testing}

The test will be carried out 6 times with the RMSE value taken based on the average RMSE value. The range of variable of acceleration values was tested from 1 to 20 . The research conducted by Anggodo \& Cholissodin [29] get the best score of 7 in that range. Fig. 8 shows the results of the variable of the acceleration test.

Fig. 8 shows that the results achieved are very fluctuating, making it difficult for the writer to get the best score. From the values of 1 to 5 increase

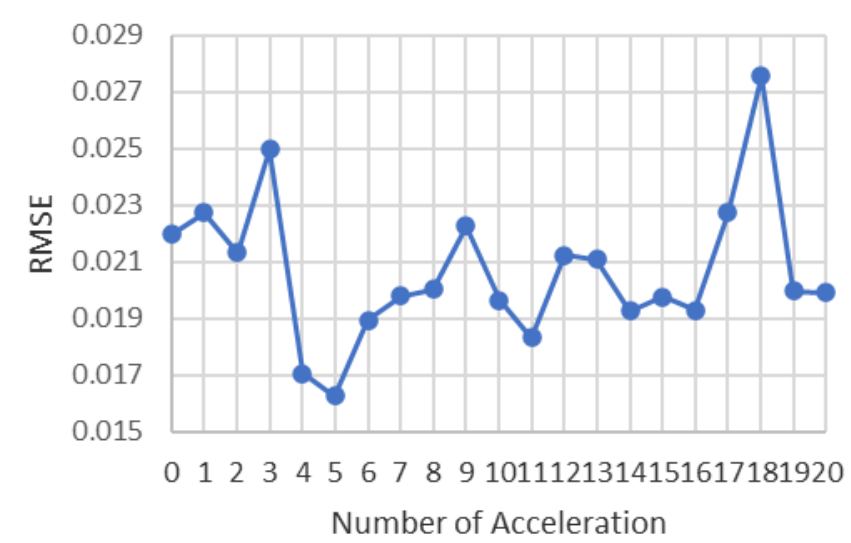

Figure. 8 Result of the variable of acceleration testing

and get the lowest value at point 5 then experience a very significant increase at point 9 and go back down at points 10 and 11 , after that it increases again and decreases again to point 19 and 20 then convergent conditions occur (there is no change in value at any point).

The test ends at point 20 because at that point it does not get a lower value as in point 5 . This test aims to find out how fast the speed changes, which results in the displacement of position on the particle [29]. In this test, the variable of acceleration is set to 5 which has an RMSE value of 0.016245829 with computation time of 18 seconds. Computation time for all tests from $r$ values of 0 to 20 requires computation time between $16-24$ seconds. This is the advantage of the auto-speed acceleration algorithm method, which has a low RMSE value with a short computing time.

\subsection{Number of time testing $(t)$}

This test aims to determine the optimal time used by the particle in carrying out displacement. Time testing is related to the acceleration test (a) because $a$ is a change in speed divided by the difference in time [22]. Figure 9 shows the result of time.

Fig. 9 shows that the time needed to reach the convergence point is 10 . The relationship between acceleration and time is an inverse comparison, the time value needed is smaller than the value of

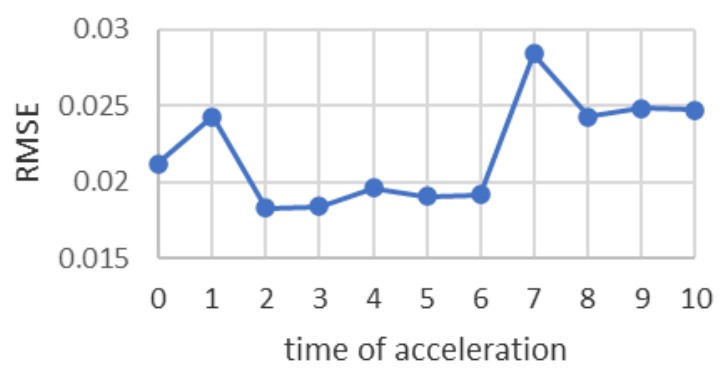

Figure. 9 Result of time testing 
acceleration and vice versa. This time test is intended to find out how much time is used for particles to carry out displacement. Because this method refers to the concept of one-dimensional motion so that the time to move is calculated. In this test, the value of time is 2 which has the smallest error value of 0.018280953 compared to the overall test.

Based on the tests, the authors determine the architectural model for forecasting the inflation rate in Indonesia by using the auto-speed acceleration algorithm, namely the number of particles is 100 , iterates a number of 110 , inertia weight 0.8 , values $c 1$ and $c 2=2$, the number of hidden neurons 3 , the acceleration is 5 and number of times is 2 with the RMSE value of 0.019261 computing time of 17 seconds.

\subsection{Comparison work}

To prove the effectiveness the proposed method for inflation forecasting in Indonesia, several comparison methods including backpropagation training methods, extreme learning machine (ELM), ELM optimized using genetic algorithms, optimized ELM using particle swarm optimization are tested. Table 2 shows the results of comparison of the overall methods. The results are averaged from 5 runs.

Table 2 shows that the ELM provides better result (lower RMSE) than the backpropagation neural network. It proves the effectiveness of ELM for forecasting. It is possible that the backpropagation neural network produces better results. However, a high computational time is required. In contrast, the ELM requires only one iteration to adjust its weight for more accurate result.

Table 2 shows that the hybrid ELM and genetic algorithm does not provide a significant better result than the ELM. Thus, improving the ELM using the genetic algorithm is not effective. The PSO can be used to improve the performance of the ELM. Table2 shows that the hybrid ELM and PSO

Table 2. Comparison result

\begin{tabular}{|c|c|c|c|c|}
\hline Methods & $\begin{array}{c}\text { Neu } \\
\text { rons }\end{array}$ & $\begin{array}{c}\text { Iterati } \\
\text { on }\end{array}$ & RMSE & $\begin{array}{c}\text { Time } \\
\text { seconds }\end{array}$ \\
\hline $\begin{array}{c}\text { Backpropag } \\
\text { ation [26] }\end{array}$ & 70 & 900 & 1.16036 & 5 \\
\hline ELM [13] & 20 & 1 & 0.02321 & 0 \\
\hline $\begin{array}{c}\text { GA-ELM } \\
{[14]}\end{array}$ & 4 & 300 & 0.02308 & 65 \\
\hline $\begin{array}{c}\text { PSO-ELM } \\
{[14]}\end{array}$ & 3 & 110 & 0.02020 & 5 \\
\hline $\begin{array}{c}\text { AUTO- } \\
\text { ELM }\end{array}$ & 3 & 110 & 0.01926 & 17 \\
\hline
\end{tabular}

provides a significant better result than the ELM. Thus, it shows that the PSO could provide better initial weights for ELM to obtain more accurate forecasting results.

The main finding of this study is the auto speed acceleration algorithm could improve the performance of particles in the PSO to search better solution. The solution is better initial weights of the ELM that can be used to produce more accurate forecasting results. The hybridization of PSO, auto speed acceleration algorithm, and ELM (AUTOELM) produce an RMSE of of 0.01926, it is the smallest error than others. The ELM method gets the smallest time computations because the method only has one iteration and the resulting value is erratic sometimes high or low depending on the value of the initial weight. To address these weaknesses, the hybrid AUTO-ELM method is used, whose performance is better than the basic ELM, because in the proposed method the weight used has been optimized using the auto-speed acceleration algorithm.

\section{Conclusion}

Inflation is one of the important indicators in analysing the economy of a country because it has a broad impact and is closely related to aggregate macroeconomic variables. Based on the previous research, it can be determined that the variables that influence the inflation rate significantly include the consumer price index, exchange rate, money supply, interest rate, and gross domestic product such as credit value and asset value.

The main finding of this study is the auto speed acceleration algorithm could improve the performance of particles in the PSO to search better solution. The solution is better initial weights of the ELM that can be used to produce more accurate forecasting results. To measure the accuracy of the proposed method, we employ comparison methods including backpropagation, extreme learning machine (ELM), hybrid ELM and genetic algorithms, Hybrid ELM and particle swarm optimization. The computational experiments shows that the proposed approach produces RMSE of 0.01926 and outperforms all comparison methods.

Other finding is the best parameter values for the hybrid ELM architecture model and auto speed acceleration algorithm, consist of the number of particles of 100 , iterations of 110 , inertia weight 0.8 , values of $c 1$ and $c 2=2$, number of hidden neurons of 3 , and acceleration value of 5 . 


\section{Conflicts of Interest}

The authors declare no conflict of interest.

\section{Author Contributions}

Conceptualization, Wayan Firdaus Mahmudy, Adyan Nur Alfiyatin and Candra Fajri Ananda; methodology, Wayan Firdaus Mahmudy; Adyan Nur Alfiyatin, and Candra Fajri Ananda; software, Adyan Nur Alfiyatin; validation, Wayan Firdaus Mahmudy and Agus Wahyu Widodo; writingoriginal draft preparation, Adyan Nur Alfiyatin and Wayan Firdaus Mahmudy; writing - review and editing, Wayan Firdaus Mahmudy, Adyan Nur Alfiyatin and Agus Wahyu Widodo.

\section{References}

[1] N. G. Mankiw, Macroeconomics fifth edition, 9 edition. New York: Worth Publishers, 2010.

[2] D. P. Rakyat, "Bank Indonesia", UndangUndang Republik Indonesia, pp. 1-12 2004.

[3] A. Kadir, P. R. Widodo, and G. R. Suryani, The Implementation of Monetary Policy in the Framework of Inflation Targeting in Indonesia (Penerapan Kebijakan Moneter dalam Kerangka Inflation Targeting di Indonesia), 2008.

[4] P. Purnawansyah, H. Haviluddin, R. Alfred, and A. F. O. Gaffar, "Network traffic time series performance analysis using statistical methods", Knowledge Engineering and Data Science, Vol. 1, No. 1, pp. 1-7, 2017.

[5] D. A. Verano, H. Husnawati, and E. Ermatita, "Implementation of Autoregressive Integrated Moving Average Model to Forecast Raw Material Stock in The Digital Printing Industry", Journal of Information Technology and Computer Science, Vol. 5, No. 1, pp. 13-22, 2020.

[6] A. C. Petrică, S. Stelian, and A. Tindeche, "Limitation of ARIMA models in financial and monetary economics", Theoretical and Applied Economics, Vol. 23, No. 4, pp. 19-42, 2016.

[7] V. Meilia, B. D. Setiawan, and N. Santoso, "Extreme Learning Machine Weights Optimization Using Genetic Algorithm In Electrical Load Forecasting", Journal of Information Technology and Computer Science, Vol. 3, No. 1, pp. 77-87, 2018.

[8] S. Chandar, S. Mahadevan, and S. N. Sivanandam, "Forecasting of foreign currency exchange rate using neural network", International Journal of Engineering and Technology, Vol. 7, No. 1, pp. 99-108, 2015.
[9] G. C. Silva, J. L. R. Silva, A. C. Lisboa, D. A. G. Vieira, and R. R. Saldanha, "Advanced fuzzy time series applied to short term load forecasting", In: Proc. of 2017 IEEE Latin American Conference on Computational Intelligence, pp. 1-6, 2017.

[10] N. R. Sari, W. F. Mahmudy, and A. P. Wibawa, "Backpropagation on Neural Network Method for Inflation Rate Forecasting in Indonesia", International Journal Advance Soft Computing Application, Vol. 8, No. 3, pp. 70-87, 2016.

[11] N. R. Sari, W. F. Mahmudy, A. P. Wibawa, and E. Sonalitha, "Enabling External Factors for Inflation Rate Forecasting using Fuzzy Neural System", International Journal of Electrical and Computer Engineering (IJECE), Vol. 7, No. 5, pp. 2746-2756, 2017.

[12] J. M. Pinto and E. F. Marcal, "Inflation Rate Forecasting: Extreme Learning Machine as a Model Combination Method", Theory and Applications of Time Series Analysis, pp. 365385, 2019.

[13] A. N. Alfiyatin, W. F. Mahmudy, C. F. Ananda, and Y. P. Anggodo, "Application of Extreme Learning Machine for Predicting Inflation Rate in Indonesia (Penerapan Extreme Learning Machine untuk Peramalan Laju Inflasi di Indonesia)", Jurnal Teknologi Informasi dan Ilmu Komputer (JTIIK), Vol. 6, No. 2, pp. 179186, 2019.

[14] A. N. Alfiyatin, A. M. Rizki, W. F. Mahmudy, and C. F. Ananda, "Extreme learning machine and particle swarm optimization for inflation forecasting", International Journal of Advanced Computer Science and Applications, Vol. 10, No. 4, pp. 473-478, 2019.

[15] N. R. Sari, W. F. Mahmudy, and A. P. Wibawa, "The effectiveness of hybrid backpropagation Neural Network model and TSK Fuzzy Inference System for inflation forecasting", Journal of Telecommunication, Electronic and Computer Engineering, Vol. 9, No. 2, pp. 111117, 2017.

[16] Y. P. Anggodo and I. Cholissodin, "Improve Interval Optimization of FLR using Auto-Speed Acceleration Algorithm", Telecomunication, Computing Electronics and Control (TEKOMNIKA), Vol. 16, No. 1, pp. 1-12, 2017.

[17] W. Sun, C. Wang, and C. Zhang, "Factor analysis and forecasting of $\mathrm{CO} 2$ emissions in Hebei, using extreme learning machine based on particle swarm optimization", Journal of Cleaner Production, Vol. 162, pp. 1095-1101, 2017.

[18] A. M. Hasan, A. G. Mahmoud, and Z. M. 
Hasan, "Optimized Extreme Learning Machine for Forecasting Confirmed Cases of COVID19", International Journal of Intelligent Engineering and Systems, Vol. 14, No. 2, pp. 484-494, 2021.

[19] S. Kamley, S. Jaloree, and R. S. Thakur, "Performance forecasting of share market using machine learning techniques: A review", International Journal of Electrical and Computer Engineering, Vol. 6, No. 6, pp. 31963204, 2016.

[20] S. Sa'adah, T. H. Liong, and Adiwijaya, "Prediction system of economic crisis in Indonesia using time series analysis and system dynamic optimized by genetic algorithm," In: Proc. of International Conference on System Engineering and Technology (ICSET), pp. 1-6, 2012.

[21] M. G. Nababan, Y. P. Anggodo, and R. R. M. Putri, "Belief Value Development In DempsterShafer With Particle Swarm Optimization(PSO) for Determining of the Provision on Cases of Persecution", Journal of Information Technology and Computer Science, Vol. 2, No. 2, pp. 57-64, 2017.

[22] D. C. Giancoli, Physics (Principles with Applications), Vol. 84, United States of America: Pearson Education, 1900.

[23] J. Kennedy and R. Eberhart, "Particle swarm optimization", In: Proc. of 1995 IEEE International Conference on Neural Networks (ICNN 95), Vol. 4, pp. 1942-1948, 1995.

[24] G. B. Huang, Q. Y. Zhu, and C. K. Siew, "Extreme learning machine: a new learning scheme of feed forward neural networks", In: Proc. of 2004 IEEE International Joint Conference on Neural Networks (IEEE Cat. No.04CH37541), Vol. 2, pp. 25-29, 2004.

[25] G. B. Huang, Q. Y. Zhu, and C. K. Siew, "Extreme learning machine: Theory and applications", Neurocomputing, Vol. 70, No. 13, pp. 489-501, 2006.

[26] A. Rahmi, V. N. Wijayaningrum, W. F. Mahmudy, and A. M. A. K. Parewe, "Offline signature recognition using back propagation neural network", Indonesian Journal of Electrical Engineering and Computer Science, Vol. 4, No. 3, 2016.

[27] W. F. Mahmudy, M. Z. Sarwani, A. Rahmi, and A. W. Widodo, "Optimization of Multi-Stage Distribution Process Using Improved Genetic Algorithm", International Journal of Intelligent Engineering and Systems, Vol. 14, No. 2, pp. 211-219, 2021.

[28] I. Cholissodin and R. K. Dewi, "Optimization of Healthy Diet Menu Variation using PSOSA", Journal of Information Technology and Computer Science, Vol. 2, No. 1, pp. 28-40, 2017.

[29] Y. P. Anggodo and I. Cholissodin, "Improve Interval Optimization of FLR using Auto-speed Acceleration Algorithm", Telecommunication Computing Electronics and Control (TELKOMNIKA), Vol. 16, No. 4, p. 1724, 2018.

[30] R. C. Eberhart and Y. Shi, "Comparing inertia weights and constriction factors in particle swarm optimization", IEEE Congress on Evolutionary Computation, Vol. 1, No. 7, pp. 84-88, Vol. 1, 2000.

[31] C. Dong, Z. Chen, and S. Sun, "The Acceleration Coefficients Self-Adapting In PSO", International Journal of Digital Content Technology and its Applications, Vol. 7, No. 5, pp. 672-678, 2013.

[32] I. Cholissodin and E. Riyandani, Swarm Intelligence, Malang: Universitas Brawijaya, 2016.

[33] M. Chandra, C. Utomo, W. F. Mahmudy, and S. Anam, "Determining the neuron weights of fuzzy neural networks using multi-populations particle swarm optimization for rainfall forecasting", Journal of Telecommunication, Electronic and Computer Engineering, Vol. 9, No. 2-8, 2017.

[34] Y. Huang and D. Lai, "Hidden Node Optimization for Extreme Learning Machine", In: Proc. of AASRI Conference on Modelling, Identification and Control, Vol. 3, pp. 375-380, 2012. 